\title{
COVID-19, Diabetes and Steroids: The Demonic Trident for Mucormycosis
}

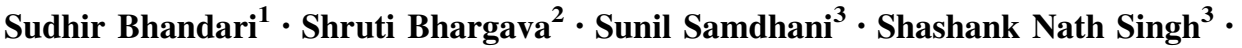 \\ Bharat Bhushan Sharma $^{1}$ - Sunita Agarwal $^{3}$ - Man Prakash Sharma ${ }^{3}$. \\ Srikant Sharma ${ }^{1} \cdot$ Vishnu Sharma $^{1} \cdot$ Shivankan Kakkar $^{4}$ - Amitabh Dube ${ }^{5}$. \\ Mohnish Grover ${ }^{3}$ (D)
}

Received: 18 August 2021/Accepted: 18 September 2021/Published online: 4 October 2021

(C) Association of Otolaryngologists of India 2021

\begin{abstract}
Coronavirus disease 2019 (COVID-19) has been found to be associated with mucormycosis in few parts of the world, especially India. It is important to look for reasons for this upsurge of cases so that other countries may take proper steps to prevent it. A prospective clinicodemographic study was conducted in SMS Medical College, Jaipur, India from April to May 2021. All patients (235) with COVID associated mucormycosis (CAM) were studied in detail with reference to their diabetic status and steroid intake during treatment of COVID-19. Steroid usage was in $84.3 \%$ of patients with methylprednisolone being the most commonly used steroid $(66.8 \%)$. Majority of the patients had taken steroids for 7-14 days. Diabetes was found in 204 patients and $42.1 \%$ of patients were newly diagnosed during/after COVID-19 treatment. The $\mathrm{HbA1c}$ levels of diabetic patients ranged from $6.0 \%$ to $16.3 \%$. This is perhaps the biggest study in the world shows that the triad of COVID-19, diabetes and steroid usage is a major contributing factor towards mucormycosis. Proper steps should be taken to prevent CAM.
\end{abstract}

Mohnish Grover

drmohnish.aiims@gmail.com

1 Department of Medicine, SMS Medical College, Jaipur, India

2 Department of Pathology, SMS Medical College, Jaipur, India

3 Department of ENT, SMS Medical College, Jaipur, India

4 Department of Pharmacology, SMS Medical College, Jaipur, India

5 Department of Physiology, SMS Medical College, Jaipur, India
Keywords COVID associated mucormycosis . COVID 19 - Mucormycosis - Diabetes mellitus · Steroid

\section{Introduction}

COVID-19 since beginning has shown many twists and turns in its presentations, complications and sequelae [1]. Many opportunistic infections have been associated with COVID-19 [2].Mucormycosis is one of such fungal infections, which have received recent attention. It has been reported from various parts of the world, however majority of patients are from India. Mucormycosis is classified according to parts of the body involved: Rhinoorbital-cerebral (ROC), pulmonary, gastrointestinal, cutaneous and disseminated. ROC mucormycosis is commonest form of mucormycosis [3]. The first wave of COVID-19 also witnessed an upsurge of these cases and was reported in few studies, one of the biggest such series being that of Sharma et al. [4]. However, the second wave has seen a way higher increase in number of such cases.

Various reasons for this increased number of mucormycosis cases have been postulated. In this study we analyzedin detail about status of diabetes mellitus and steroid usage in CAM patients. This is probably a study with biggest sample size discussing about these 3 aspects.

\section{Material and Methods}

A prospective study was conducted from April 2021 to May 2021 at SMS Medical College, Jaipur, India, a tertiary care institute. All patients with history of COVID-19 and positive $\mathrm{KOH}$ fungal staining on nasal biopsy were taken 
up in this study. Clinico-demographic profile of the patients was studied with special reference to diabetes (known/new diabetic, HbA1c levels) and steroid usage (salt, dosage and duration). This data were then analyzed.

\section{Results}

The study involved 235 patients with confirmed CAM who were evaluated between April to May 2021. All these patients had a history of onset of symptoms of COVID-19 infection, within 45 days prior to diagnosis of mucormycosis. Eleven of these were COVID-19 positive at time of developing mucormycosis.

Out of these, 198 patients (84.3\%) had used steroids during treatment of COVID-19. The most common steroids used were methylprednisolone (66.8\%), dexamethasone (45.1\%) and prednisolone (33.6\%). Many patients had used more than one type of steroids (Table 1). $55.1 \%$ of the patients who were on steroids took oral steroids only, while the rest $44.9 \%$ had intravenous steroids followed by oral intake. Duration of steroid usage was also studied, and it was found that majority $(56.2 \%)$ of these patients took steroids for 7-14 days (Table 2).

Data regarding diabetic status of CAM patients were also gathered. A total of 204 patients had DM, $44.7 \%$ of which were known diabetics and $42.1 \%$ were newly diagnosed to be so during COVID-19 treatment (Table 3). Twenty-one $(10.3 \%)$ patients had diabetic ketoacidosis. The HbAlc levels of diabetic patients ranged from $6.0 \%$ to $16.3 \%$ (mean $10.9 \pm 3.35 \mathrm{SD}$ ).

Other co morbidities, apart from DM, encountered in our patients were hypertension in 63 patients, chronic renal disease in eight patients and three patients with malignancy.

\section{Discussion}

Phycomycosis or zygomycosis was first described in 1885 by Paltauf [5] and later coined as Mucormycosis in 1957 by Baker [6] an American pathologist for an aggressive infection caused by Rhizopus. It has since been found out that various fungi of the genus Rhizopus, Mucor, Absidia, Rhizomucor and Cunninghamella can lead to this angioinvasive disease with very high morbidity and mortality [7].

Many conditions, which lead to decreased immunity, have been known to predispose patients to mucormycosis. These include uncontrolled diabetes mellitus, solid organ transplantation, neutropenia, malignancies, iron overload, AIDS, intake of immunosuppressants, etc. [8, 9].

Mucormycosis is known to be various types depending on the site of involvement: ROC, pulmonary, gastrointestinal tract, cutaneous and disseminated, but ROCM is the most common type globally [3]. Though, ROCM is mainly a clinicoradiological diagnosis but it is important to be confirmed by microbiological/histopathological testing.

COVID-19 has seen an upsurge in cases of mucormycosis, specially in India $[10,11]$. This was seen in first wave of COVID-19 as well and a study by Sharma et al. [4] was the one of the first ones to report so. The second wave of COVID-19 has witnessed a huge increase in number of cases of ROCM. In this study, in a span of 2 months, we have encountered 235 patients of mucormycosis, and it is one of the biggest numbers of patients reported as yet of CAM from a single institute. This is a preliminary study, which was done with the aim of studying the percentage of patients of CAM having history of steroid intake during COVID-19 treatment and those with known/recent onset diabetes mellitus. John TM et al. [12] in their study on 41 patients concluded that CAM was commonly seen in patients with diabetes (94\%), especially those with poorly controlled glycemic levels (67\%). They also found that $88 \%$ has received steroids. Singh AK [13] did a review of cases of 101 cases of CAM, out of which 82 were reported from India. They found that $80 \%$ of cases had diabetes and $76.3 \%$ had taken steroids for treatment of COVID-19. In our study we found similar results, with $84.3 \%$ using steroids, most commonly used steroid being methylprednisolone with majority patients using steroid for 7-14 days. Diabetes was also frequently encountered with 204 out of 235 patients having uncontrolled glycemic levels, $44.7 \%$ being known diabetics while $42.1 \%$ were newly diagnosed to be so.

Table 1 Type of steroid used by CAM patients during COVID-19 treatment (few patients used multiple salts of steroids)

\begin{tabular}{llcc}
\hline S. no. & Type of steroids & Number of patients & Percentage $(\%)$ \\
\hline 1 & Dexamethasone & 106 & 45.1 \\
2 & Methyl prednisolone & 157 & 66.8 \\
3 & Prednisolone & 79 & 33.6 \\
4 & Others & 23 & 9.8 \\
\hline
\end{tabular}


Table 2 Duration of steroid used by CAM patients during COVID-19 treatment

\begin{tabular}{llrr}
\hline S. no. & Duration of use of steroids & Number of patients & Percentage $(\%)$ \\
\hline 1 & Less than 7 days & 75 & 31.9 \\
2 & 7 to 14 days & 132 & 56.2 \\
3 & More than 14 days & 28 & 11.9 \\
Total cases & & 235 & 100.0 \\
\hline
\end{tabular}

Table 3 Status of diabetes mellitus in patients with CAM

\begin{tabular}{lllr}
\hline S. no. & Status of diabetes & Number of patients & Percentage $(\%)$ \\
\hline 1 & New & 99 & 42.1 \\
2 & Old & 105 & 44.7 \\
3 & None & 31 & 13.2 \\
Total cases & & 235 & 100.0 \\
\hline
\end{tabular}

There could be many reasons for this triad of COVID19, Diabetes and steroid intake, predisposing to mucormycosis:

(1) COVID-19 leads to lymphopenia with reduced CD4 and CD8 counts, which leads to immunosuppression [14].

(2) Cytokine surge, particularly IL6 and IL1, leads to increased ferritin levels, cellular iron levels and serum free iron levels. Iron is required for Mucor to grow [15].

(3) Elevated ferritin level is an independent risk factor diabetes mellitus type 2 [16].

(4) COVID-19 leads to endotheliolitis, inducing a prothrombotic state and thereby thromboembolic phenomenon. Mucor is also an angioinvasive fungus. Both these have a synergistic effect [17].

(5) COVID-19 itself induces increase in blood sugar levels by its action on pancreas [18].

(6) Diabetes leads to impaired neutrophilic function adding to further immunosuppression [19].

(7) Diabetic patients with ketoacidosis, leads to decreased $\mathrm{pH}$, which does not allow binding of free iron to transferrin. Increased free iron levels predispose to mucormycosis [20].

(8) Hyperglycemia leads to glycosylation of transferrin and ferritin: reduced iron binding and increased free iron levels [21].

(9) Increased GRP 78 (Glucose Regulatory Protein 78) expression of endothelial cells and fungal ligand spore coating homolog $(\mathrm{CotH})$ protein: This mediates angioinvasion and damage of human endothelial cells by the fungi [22].
(10) Steroids are known to have multiple immunosuppression effects[23]. They are also known to lead to hyperglycemia [24].

The authors also feel that this variant of COVID-19 (delta variant) could be a reason for susceptibility to mucormycosis.

All the above reasons, to a varying degree, have contributed to the increased incidence of mucormycosis. It is important that treatment should therefore be aimed at handling all these issues.

\section{Conclusion}

This study with a big sample size, probably the biggest in the world literature, emphasizes on the demonic triad of COVID-19, diabetes and steroids. Immunosuppressive effect of COVID-19 cannot be altered, but it is important that doctors and general public be made aware about judicial steroid intake for treatment of COVID-19 and control of blood sugar levels. Just like this unholy triad leading to CAM, the three-pronged treatment consisting of glycemic control, antifungal and surgical debridement need to be started simultaneously.

Funding None.

Availability of data and material Yes.

Code availability None.

Declarations 
Conflicts of interest The authors declared that they have no conflict of interst.

\section{Ethical Approval Taken.}

Consent to Participate Yes.

Consent for Publication Yes.

\section{References}

1. Haleem A, Javaid M, Vaishya R (2020) Effects of COVID-19 pandemic in daily life. Curr Med Res Pract 10(2):78-79

2. Kubin CJ, McConville TH, Dietz D, Zucker J, May M, Nelson B et al (2021) Characterization of bacterial and fungal infections in hospitalized patients with COVID-19 and factors associated with healthcare-associated infections. Open Forum Infect Diss 8:ofab201

3. Jaju MR, Sagar RV, Srivastav SK, Singh R, Kumar KV, Faisal M (2020) Different types of mucormycosis: case series. Int J Res Med Sci 8(6):2284-2296

4. Sharma S, Grover M, Bhargava S, Samdani S, Kataria T (2021) Post coronavirus disease mucormycosis: a deadly addition to the pandemic spectrum. J Laryngol Otol 8:1-6

5. Paltauf A (1885) Mycosis mucorina: Ein Beitrag zur Kenntniss der menschlichen Fadenpilzerkrankungen. Arch Für Pathol Anat Physiol Für Klin Med 102(3):543-564

6. Baker RD (1957) Mucormycosis; a new disease? J Am Med Assoc 163(10):805-808

7. Eucker J, Sezer O, Graf B, Possinger K (2001) Mucormycoses. Mycoses 44(7-8):253-260

8. de Shazo RD (1998) Fungal sinusitis. Am J Med Sci 316(1):39-45

9. Sugar AM (1992) Mucormycosis. Clin Infect Dis Off Publ Infect Dis Soc Am 14(Suppl 1):S126-129

10 Moorthy A, Gaikwad R, Krishna S, Hegde R, Tripathi KK, Kale PG et al (2021) SARS-CoV-2, uncontrolled diabetes and corticosteroids-An unholy trinity in invasive fungal infections of the maxillofacial region, a retrospective multi-centric analysis. J Maxillofac Oral Surg. https://doi.org/10.1007/s12663-021-015 32-1

11. Sarkar S, Gokhale T, Choudhury SS, Deb AK (2021) COVID-19 and orbital mucormycosis. Indian J Ophthalmol 69(4):1002-1004

12 John TM, Jacob CN, Kontoyiannis DP (2021) When uncontrolled diabetes mellitus and severe COVID-19 converge: the perfect storm for mucormycosis. J Fungi Basel Switz. 7(4):298
13. Singh AK, Singh R, Joshi SR, Misra A (2021) Mucormycosis in COVID-19: a systematic review of cases reported worldwide and in India. Diabetes Metab Syndr Clin Res Rev [Internet]. 2021 May 21 [cited 2021 May 31]; Available from: https://www. sciencedirect.com/science/article/pii/S1871402121001570

14. Tavakolpour S, Rakhshandehroo T, Wei EX, Rashidian M (2020) Lymphopenia during the COVID-19 infection: What it shows and what can be learned. Immunol Lett 225:31-32

15. Iwasaki M, Saito J, Zhao H, Sakamoto A, Hirota K, Ma D (2021) Inflammation triggered by SARS-CoV-2 and ACE2 augment drives multiple organ failure of severe COVID-19: molecular mechanisms and implications. Inflammation 44(1):13-34

16. Liu J, Li Q, Yang Y, Ma L (2020) Iron metabolism and type 2 diabetes mellitus: a meta-analysis and systematic review. J Diabetes Investig 11(4):946-955

17. Perico L, Benigni A, Casiraghi F, Ng LFP, Renia L, Remuzzi G (2020) Immunity, endothelial injury and complement-induced coagulopathy in COVID-19. Nat Rev Nephrol 19:1-19

18. Kusmartseva I, Wu W, Syed F, Van Der Heide V, Jorgensen M, Joseph P et al (2020) Expression of SARS-CoV-2 entry factors in the pancreas of normal organ donors and individuals with COVID-19. Cell Metab 32(6):1041-1051.e6

19. Alba-Loureiro TC, Munhoz CD, Martins JO, Cerchiaro GA, Scavone C, Curi R et al (2007) Neutrophil function and metabolism in individuals with diabetes mellitus. Braz J Med Biol Res Rev Bras Pesqui Medicas E Biol 40(8):1037-1044

20. Artis WM, Fountain JA, Delcher HK, Jones HE (1982) A mechanism of susceptibility to mucormycosis in diabetic ketoacidosis: transferrin and iron availability. Diabetes 31(12):1109-1114

21. Baldin C, Ibrahim AS (2021) Molecular mechanisms of mucormycosis-The bitter and the sweet. PLoS Pathog [Internet]. 2017 Aug 3 [cited 2021 May 31];13(8). Available from: https://www.ncbi.nlm.nih.gov/pmc/articles/PMC5542377/

22. Gebremariam T, Liu M, Luo G, Bruno V, Phan QT, Waring AJ et al (2014) CotH3 mediates fungal invasion of host cells during mucormycosis. J Clin Invest 124(1):237-250

23. Barshes NR, Goodpastor SE, Goss JA (2004) Pharmacologic immunosuppression. Front Biosci J Virtual Libr 1(9):411-420

24. Tamez-Pérez HE, Quintanilla-Flores DL, Rodríguez-Gutiérrez R, González-González JG, Tamez-Peña AL (2015) Steroid hyperglycemia: Prevalence, early detection and therapeutic recommendations: a narrative review. World $\mathrm{J}$ Diabetes 6(8):1073-1081

Publisher's Note Springer Nature remains neutral with regard to jurisdictional claims in published maps and institutional affiliations. 